\title{
Linear IGA Bullous Dermatosis Following Influenza Vaccination; A Coincidence?
}

\section{Qasem NA*, Atia $\mathrm{H}$ and Al Otaibi MF}

Dermatology Department, Al-Jahra'a Hospital, Ministry of Health, Kuwait

*Corresponding author: Naser Qasem, State of Kuwait, Qurtoba, Postal code 73501, Kuwait, Tel: 00965 95565157; Email: Naserqasem@outlook.com

\section{Case Report}

Volume 5 Issue 4

Received Date: October 27, 2020

Published Date: November 13, 2020

DOI: $10.23880 /$ cdoaj-16000224

\section{Abstract}

Background: Linear immunoglobulin A (IgA) bullous dermatosis (LABD) is a rare, immune-mediated blistering disorder characterised by the deposition of a linear band of IgA at the dermoepidermal junction. The exact cause and triggering factors of LABD still remain unknown.

Main observations: A previously healthy 40-year-old male developed widespread vesico-bullous lesions shortly after receiving influenza vaccination. A punch biopsy obtained from one of the lesions revealed a diagnosis of LABD.

Conclusion: Further research needs to be done in order to establish the underlying triggers for LABD. This case report demonstrates that a causal relationship between influenza vaccination and the initiation of LABD may exist in susceptible individuals.

Keywords: Bullous Disease; Vaccination; Immunization; Influenza; Linear IgA Bullous Disease

\section{Introduction}

Linear immunoglobulin A (IgA) bullous dermatosis (LABD) is a rare, immune-mediated blistering disorder characterised by the deposition of a linear band of IgA at the dermoepidermal junction [1]. The clinical presentation of LABD involves lesions on the skin, mucous membranes or both. The pathogenesis of LABD seems to involve both cellular and immune responses, which result in tissue injury secondary to induction of inflammation via circulating autoantibodies and the release of proteolytic enzymes from inflammatory cells [2]. Target antigens in LABD include $97 \mathrm{kDa}$ and $120 \mathrm{kDa}$, which are residuals of the bullous pemphigoid antigen 2 (BP180/type XVII collagen), a transmembrane protein that plays an important role in dermal-epidermal adhesion and the structural integrity of the basement membrane zone $[3,4]$.

\section{Case Report}

\section{Patient History}

A previously healthy 40-year-old Arab man presented to our dermatology department two months after a rapid eruption of severely pruritic vesico-bullous lesions on numerous sites on his body. This started two weeks after receiving vaccinations for pneumococcal disease, meningococcal disease and influenza. There is no personal history of skin disease, however, both the patient's mother and daughter have vitiligo.

\section{Clinical Appearance}

Multiple small, scattered, crusted erosions were noted on the neck, upper trunk (Figure 1), extensor aspect of both upper and lower extremities, groin, scrotum (Figure 2) and mouth (Figure 3), with few intact blisters. 


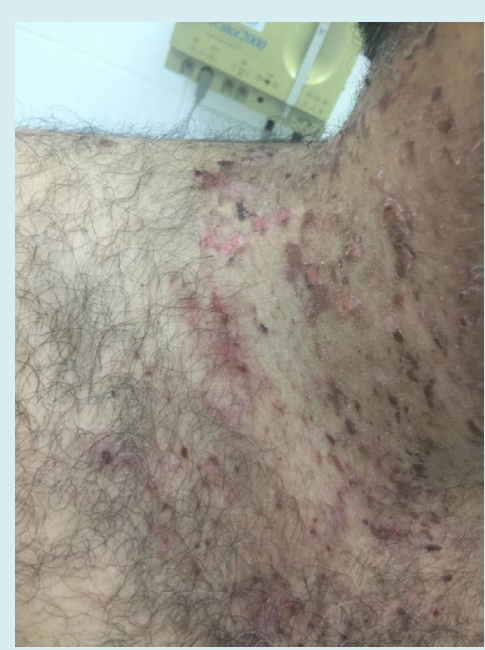

Figure 1: A close-up perspective on the patient's skin lesions. As his condition is severely pruritic, note that the illustrated skin lesions are sites of previously intact bullae and vesicles.
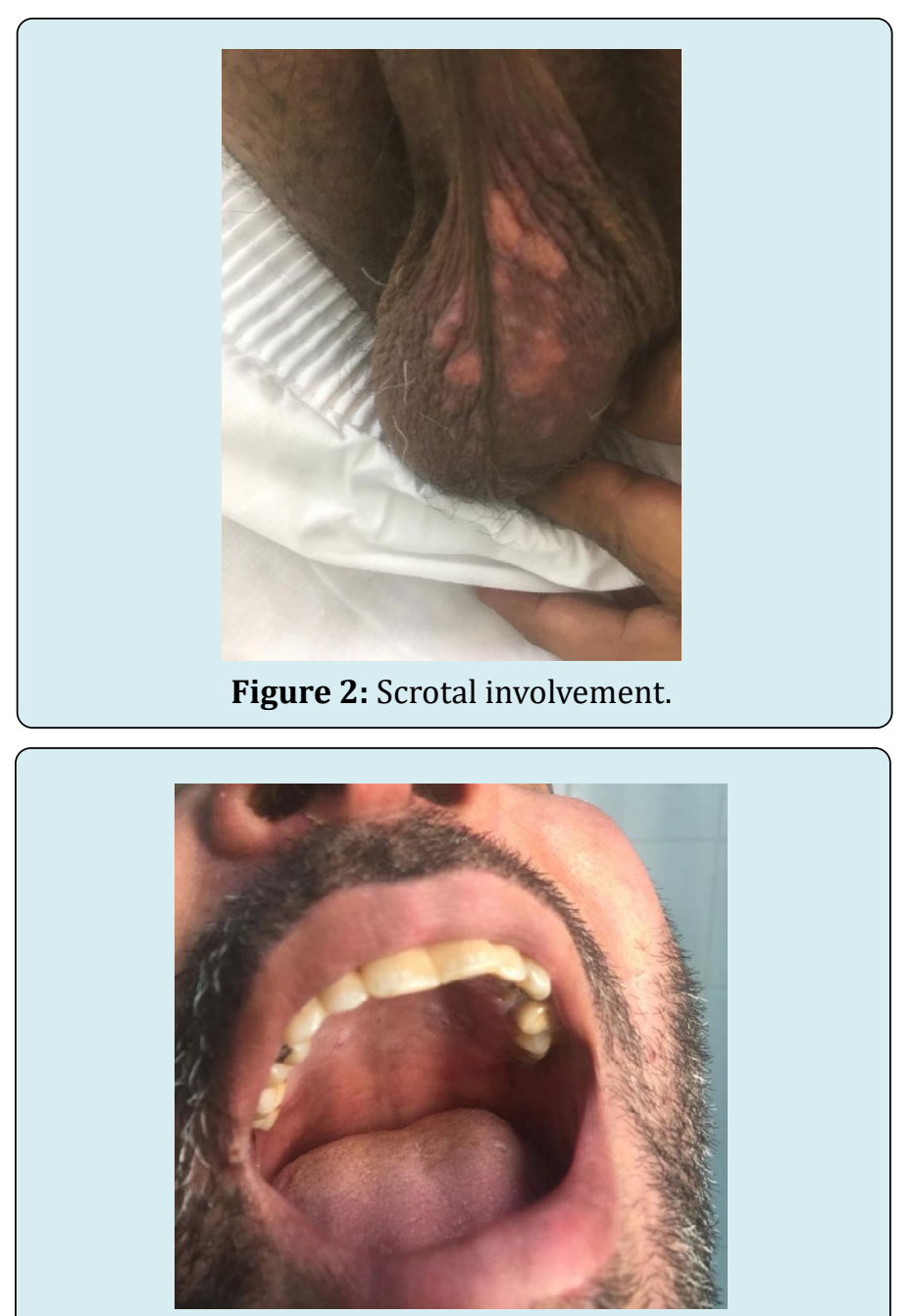

Figure 3: Oral mucosal involvement.

\section{Histopathology Report}

Subepidermal blister formations with papillary dermal oedema were evident. The blister cavity contained sero-exudate and neutrophils. In the dermis, there was a perivascular mixture of inflammatory cells, including lymphocytes, eosinophils and neutrophils. The direct immunofluorescence showed the characteristic linear band of IgA in LABD.

\section{Discussion}

The significance of this case report stems from several factors. First, LABD is a rare disorder [5]. Its incidence varies across countries, and reports of its incidence have fluctuated from less than 0.6 to 2.2 cases per million individuals per year [2]. Second, and most importantly, the exact cause of linear IgA disease remains unknown [6]. This autoimmune condition can either have an idiothetic presentation or be triggered by exposure to certain drugs [1]. Vancomycin has been heavily reported as a potential provoker of LABD [6].

A salient fact of our patient's history is that he received three vaccinations two weeks prior to his disease eruption. In the literature, there are several case reports of vaccinations inducing autoimmune blistering disorders $[7,8]$. One study reported the case of a 3-month-old boy who developed severe blistering symptoms a couple of days after receiving a combined vaccination against hepatitis B, pertussis, poliomyelitis, diphtheria, tetanus and haemophilus influenzae. The same study also described the case of a 17-month-old girl who developed blistering symptoms one week after receiving the previously mentioned combined vaccination [7]. Moreover, it has also been reported that the human papilloma vaccination is associated with LABD [8].

Of the three vaccinations our patient received, the influenza vaccination stands out. This vaccination has been identified as an initiator of autoimmune phenomena [9]. Numerous reports of new-onset bullous pemphigoid (BP) have been temporally correlated with influenza immunisation [10-13]. Other reports have demonstrated that immunisation with influenza exacerbates pre-existing BP [12]. The time interval from vaccination to onset of skin lesions ranged from one day to less than one month in all the studies mentioned above. In another case study, recurrence of bullae and erosions was noted seven days after a patient with pemphigus vulgaris in remission received influenza immunisation. It was noted that the same patient had a similar reactivation, with a similar latency period, years earlier, also after receiving influenza immunisation [14]. Nondermatological autoimmune reactions, especially cardiac, neurologic, hematologic and rheumatic, have also been thought to be induced by influenza immunisation. Although these autoimmune manifestations were largely self-limited, 


\section{Clinical Dermatology Open Access Journal}

most of them occurred within one month of influenza immunisation [15]. The precise mechanism of autoimmune manifestations following influenza immunisation remains undetermined $[15,16]$. A plausible hypothesis is that certain host antigens structurally resemble viral antigens, a phenomenon known as molecular mimicry [16]. Another explanation is the indirect and direct activation of the host's immune system by cytokines and viral antigens, respectively [15]. There has been only one case report of a patient who developed LABD two days after receiving influenza vaccination (the patient was a 54-year-old woman) [9]. Thus, it is reasonable to suggest that the influenza vaccination may have been, directly or indirectly, the trigger.

A two-week interval between the immunisation and the onset of skin lesions might be adduced as an argument against the presence of a causal relationship between them. However, in drug-induced LABD, the disease commonly commences one month after initial drug exposure [17-19]. Moreover, our patient has a significant family history of an autoimmune condition (vitiligo). Evidence has demonstrated that an exposure to a known provocative drug can lead to a more severe and rapid reappearance of blistering [20]. Hence, if the vaccinations were in fact the cause of our patient's condition, it is essential to establish a causal relation, as this may prevent further and future disease eruptions.

\section{Conclusion}

We discuss the case of a patient who developed an eruption of bullous two weeks after receiving an influenza vaccination. Although the exact mechanism of induction of his condition is uncertain, the recognition that influenza vaccination initiates autoimmune phenomena, the short time interval between the patient's immunisation and onset of his lesions and one report of a similar case in the literature indicate that there may be an association between influenza vaccination and LABD initiation.

\section{References}

1. Lawley TJ, Strober W, Yaoita H, Katz SI (1980) Small intestinal biopsies and HLA types in dermatitis herpetiformis patients with granular and linear IgA skin deposits. J Invest Dermatol 74(1): 9-12.

2. Fortuna G, Marinkovich MP (2012) Linear immunoglobulin A bullous dermatosis. Clin Dermatol 30(1): 38-50.

3. Guide SV, Marinkovich MP (2001) Linear IgA bullous dermatosis. Clin Dermatol 19(6): 719-727.

4. Chorzelski TP, Jablonska S, Maciejowska E (1991) Linear IgA bullous dermatosis of adults. Clin Dermatol 9(3):
383-392.

5. Mintz EM, Morel KD (2011) Clinical features, diagnosis, and pathogenesis of chronic bullous disease of childhood. Dermatol Clin 29(3): 459-62.

6. Fortuna G, Salas-Alanis JC, Guidetti E, Marinkovich MP (2012) A critical reappraisal of the current data on druginduced linear immunoglobulin A bullous dermatosis: a real and separate nosological entity? J Am Acad Dermatol 66(6): 988-994.

7. Baroero L, Coppo P, Bertolino L, Maccario S, Savino F (2017) Three case reports of post immunization and post viral Bullous Pemphigoid: looking for the right trigger. BMC Pediatr 17(1): 60.

8. Ikeya S, Urano S, Tokura Y (2012) Linear IgA bullous dermatosis following human papillomavirus vaccination. Eur J Dermatol 22(6): 787-788.

9. Alberta-Wszolek L, Mousette AM, Mahalingam M, Levin NA (2009) Linear IgA bullous dermatosis following influenza vaccination. Dermatol Online J 15(11): 3.

10. Fournier B, Descamps V, Bouscarat F, Crickx B, Belaich S (1996) Bullous pemphigoid induced by vaccination. $\mathrm{Br} \mathrm{J}$ Dermatol 135(1): 153-154.

11. Lear JT, Tan BB, English JS (1996) Bullous pemphigoid following influenza vaccination. Clin Exp Dermatol 21(5): 392 .

12. Downs AM, Lear JT, Bower CP, Kennedy CT (1998) Does influenza vaccination induce bullous pemphigoid? A report of four cases. Br J Dermatol 138(2): 363.

13. Nikkels AF, Nikkels-Tassoudji N, Pierard GE (2005) Cutaneous adverse reactions following anti-infective vaccinations. Am J Clin Dermatol 6(2): 79-87.

14. De Simone C, Caldarola G, D'Agostino M, Zampetti A, Amerio P, et al. (2008) Exacerbation of pemphigus after influenza vaccination. Clin Exp Dermatol 33(6): 718720.

15. Schattner A (2005) Consequence or coincidence? The occurrence, pathogenesis and significance of autoimmune manifestations after viral vaccines. Vaccine 23(30): 3876-3886.

16. Shoenfeld Y, Aron-Maor A (2000) Vaccination and autoimmunity-'vaccinosis': a dangerous liaison? J Autoimmun 14(1): 1-10.

17. Ho JC, Ng PL, Tan SH, Giam YC (2007) Childhood linear IgA bullous disease triggered by amoxicillin-clavulanic 
Clinical Dermatology Open Access Journal

acid. Pediatr Dermatol 24(5): E40-E43.

18. Nousari HC, Costarangos C, Anhalt GJ (1998) Vancomycinassociated linear IgA bullous dermatosis. Ann Intern Med 129(6): 507-508.
19. Navi D, Michael DJ, Fazel N (2006) Drug-induced linear IgA bullous dermatosis. Dermatol Online J 12(5): 12.

20. Whitworth JM, Thomas I, Peltz SA, Sullivan BC, Wolf AH, et al. (1996) Vancomycin-induced linear IgA bullous dermatosis (LABD). J Am Acad Dermatol 34(5): 890-891. 